\title{
Effects of anesthesia on conventional and speckle tracking echocardiographic parameters in a mouse model of pressure overload
}

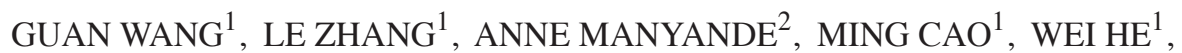 \\ XIAOFEN WU ${ }^{1}$, JINLI WANG ${ }^{1}$ and CUNTAI ZHANG ${ }^{1}$ \\ ${ }^{1}$ Department of Gerontology, Tongji Hospital, Tongji Medical College, Huazhong University of Science and Technology, \\ Wuhan, Hubei 430030, P.R. China; ${ }^{2}$ School of Psychology, Social Work and Human Sciences, \\ University of West London, London W5 5RF, UK
}

Received September 12, 2014; Accepted February 11, 2015

DOI: $10.3892 /$ etm.2015.2331

\begin{abstract}
Genetically-modified mice are widely applied in cardiovascular studies as model organisms. Echocardiography is a key tool for evaluating cardiac and hemodynamic functions in mice. The present study aimed to examine the effects of isoflurane (ISF) on conventional and speckle tracking echocardiography (STE) parameters under healthy and pathological conditions using a murine model of pressure overload. In addition, the optimal dose of ISF in the process of echocardiographic measurement, with minimum cardiac contraction depression, was investigated. Conventional echocardiographic and STE examinations were performed on 38 adult C57BL/6 male mice. The mice were divided into the following three groups: The sham $(n=15)$; mild thoracic aortic banding (TAB; $n=15)$; and severe TAB $(n=8)$ groups. ISF was administered under deep anesthesia (DA; 1-2\% ISF), light anesthesia (LA; $0.5-1 \%$ ISF) and immediately prior to the mice waking up (awake; 0-0.5\% ISF). Conventional echocardiographic parameters were preserved within the sham and mild TAB groups ( $\mathrm{P}>0.05$ for each parameter) under LA and awake conditions. However, under DA conditions, the majority of these parameters were reduced compared with the LA and awake conditions $(\mathrm{P}<0.05)$. In the severe TAB group, conventional echocardiographic parameters remained constant under LA, DA and awake conditions. STE parameters in the groups remained similar between the LA and awake conditions, but were significantly reduced under DA
\end{abstract}

Correspondence to: Professor Cuntai Zhang, Department of Gerontology, Tongji Hospital, Tongji Medical College, Huazhong University of Science and Technology, 1095 Jiefang Avenue, Wuhan, Hubei 430030, P.R. China

E-mail: ctzhang2000@gmail.com

Key words: mouse, heart failure, hypertrophy, echocardiography, left ventricular function conditions. Therefore, conventional echocardiography and STE may be performed using LA induced with low doses of ISF, under various pathological conditions without affecting cardiac function.

\section{Introduction}

Advancements in transgenic technology have resulted in genetically-modified mice being widely employed as model organisms in mechanistic studies of cardiovascular disease $(1,2)$. The development of accurate and non-invasive techniques for the quantitative and phenotypic characterization of cardiac function in healthy myocardium is crucial for obtaining the full benefits of these models. Echocardiography is an effective approach for quantitatively and accurately evaluating cardiac function and morphology $(3,4)$. Despite these advantages, conventional echocardiography, such as M-mode assessment, is offset by the poor sensitivity for detecting subtle cardiac dysfunction (5). Therefore, there is a constant and pressing requirement for novel echocardiography techniques to enhance the assessment of cardiac function. The deformation (strain) imaging is one of these advanced techniques, which enables the improved quantification of global and regional cardiac function. It captures segmental displacement and velocity of the left ventricle in multiple planes over the cardiac circle and can be acquired from tissue Doppler or 2-dimensional speckle tracking echocardiography (STE) (6). In the STE method, the speckles resulting from acoustic interference phenomena are used to track the movement of myocardium (7). Due to the benefits of high reproducibility and angle-independency over color tissue Doppler imagining, STE has been widely applied (8). Prior studies have demonstrated that anesthetic agents may serve a significant function in the evaluation of echocardiographic parameters in mice (9). Isoflurane (ISF) is a volatile anesthetic that has been identified as a potential anesthetic agent for use in experimental studies involving mice, as it facilitates rapid conduction and easy control of the depth of anesthesia during conventional echocardiography (10-13). 
Due to the novelty of the STE technique, few studies have examined the effects of anesthesia on the parameters of STE. Previous studies have focused primarily on investigating the effects of anesthesia on the echocardiographic analysis of healthy subjects $(9,14)$. As a result, the effects of anesthesia on cardiac function under pathological conditions remain unknown.

Therefore, the present study aimed to investigate the effects of an anesthetic agent on STE parameters in mice with hypertrophic and failing myocardium, in addition to healthy mice. Furthermore, the effects of anesthesia on conventional echocardiographic parameters were assessed in mice with healthy, hypertrophic and failing myocardium.

\section{Materials and methods}

Animals. The current study was approved by the Institutional Animal Care and Use Committee of Tongji Hospital, Huazhong University of Science and Technology (Wuhan, China). Adult C57BL/6 male mice (age, 8-10 weeks; weight, 20-24 g) were acquired from the Wuhan University Centre for Animal Experiments (Wuhan, China). Mice were maintained in an air-conditioned environment with a $12 \mathrm{~h}$ alternating light and dark cycle and received a standard rodent diet and water ad libitum.

Experimental protocol. In total, 45 mice were divided at random into the sham $(n=15)$, mild thoracic aortic banding (TAB; $n=15)$ and severe TAB $(n=15)$ groups. The mice were anesthetized with ketamine (100 mg/kg; Jiangsu Hengrui Medicine Co., Ltd., Lianyungang, China) and xylazine (5 mg/kg; Lloyd Inc., Shenandoah, IA, USA), administered intraperitoneally. A 3-mm left-sided thoracotomy was made at the second intercostal space. The transverse aortic arch was exposed and ligated (7-0 Prolene suture; Shanghai Medical Suture Needle Factory, Shanghai, China) using a 25 or $27 \mathrm{G}$ needle, between the right innominate and left common carotid arteries, to induce the mild and severe TAB, respectively. The needle was then removed rapidly, leaving a discrete region of stenosis. The chest incision was subsequently closed and the left-side pneumothorax was evacuated. The sham group underwent an identical procedure, but without the ligation of the aortic arch. At week 8, each group was examined using conventional echocardiography and STE. During the echocardiographic examinations, varying doses of ISF (Lunan Pharmaceutical Group Corporation, Shandong, China) were administered, and heart rate (HR) was used to determine the depth of the anesthesia. The three states of anesthesia that were induced in the mice were as follows: Awake (0-0.5\% ISF administered immediately prior to the mice waking up; HR, >520 bpm); light anesthesia (LA; ISF, 0.5-1\%; HR, 460-520 bpm); and deep anesthesia (DA; ISF, 1-2\%; HR, 390-460 bpm).

Anesthesia and preparation of echocardiography. The mice were anesthetized by ISF inhalation, which was administered via a Vevo Compact Dual Anesthesia System (VisualSonics, Inc., Toronto, ON, Canada). Anesthesia induction was performed for 3 min with $2 \% \mathrm{ISF} / 98 \% \mathrm{O}_{2}$. Following the failure of the righting reflex, the mice were placed on a heating pad in the supine position in order to maintain normothermia. The dose of ISF was altered as echocardiography was performed under awake, LA and DA conditions. Under each condition, the concentration of ISF was adjusted to maintain the immobility and sedation of the mice. Anesthetic was administered via a nose cone at a flow rate of $1-1.51 / \mathrm{min}$. To produce an electrocardiogram (ECG), copper electrodes on the heating pad were covered with ECG gel (Parker Laboratories, Inc., Fairfield, NJ, USA). The paws of the mice were taped to the electrodes and the ECG was recorded simultaneously. The walls of the chest were shaved and ultrasound gel (Parker Laboratories, Inc.) was applied liberally to the thoracic region to optimize the visibility of the left ventricular (LV) chamber and the wall motion.

Conventional echocardiography and parameters. Conventional echocardiography was performed using a high-resolution Vevo 2100 System with a $30 \mathrm{MHz}$ MS400 linear array transducer (VisualSonics, Inc.). B-Mode and M-mode images were obtained in short axis view at the papillary level and in parasternal long-axis view, with the aortic arch and endocardium in optimal view. The image depth, width and gain settings were adjusted during the data acquisition period. The frame rate remained at $>200 \mathrm{~Hz}$ in all $\mathrm{B}$-mode and $\mathrm{M}$-mode images in order to optimize image quality.

M-mode tracings were used to digitally measure the LV end-diastolic diameter (LVEDD), LV end-systolic diameter (LVESD) and HR. Based on these measurements, the LV end-systolic volume (LVESV) was calculated as [7.0/(2.4 + LVESD)] x LVESD. The LV end-diastolic volume (LVEDV) was calculated as [7.0/(2.4 + LVEDD)] x LVEDD. The ejection fraction (EF) was calculated as [(LVEDV-LVESV)/LVEDV] x 100\%. The fraction shortening (FS) was calculated as [(LVEDD-LVESD)/LVEDD] x $100 \%$. The stroke volume (SV) was calculated as LVEDV-LVESV. The LV mass was calculated as $1.053 \times$ [(LVEDD + LVPWTd + LVAWTd $^{3}$-LVEDD ${ }^{3}$, in which LVPWTd denotes LV posterior wall thickness in diastole and LVAWTd denotes LV anterior wall thickness in diastole. The corrected LV mass was the LV mass $x$ 0.8. All measurements were presented as the mean of three adjacent cardiac cycles.

Strain analysis. Myocardium strain analyses were performed using VevoStrain software, version 1.4.0 (VisualSonics, Inc.). Images that presented the clearest visualization of the endocardium and epicardium border with the fewest artifacts were selected. All the analyses were conducted by the same trained operator who was blinded to the groups. Tracking points were placed in three consecutive cardiac circles. The endocardium and epicardium were then traced semi-automatically (Fig. 1A and B). The tracking points were adjusted manually to achieve well-defined tracking. Images were analyzed frame-by-frame using the VevoStrain software. The left ventricles were divided into six segments. For the parasternal long-axis view, they were divided as follows: Posterior apex, posterior base, posterior medium, anterior base, anterior medium and anterior apex (Fig. 1A and C). For the short axis view, they were divided into anterior free wall, lateral wall, posterior wall, inferior free wall, posterior septum wall and anterior septum wall. Strain values and the corresponding strain-rate curves were acquired for each segment in longitudinal (Fig. 1C), radial (Fig. 1D) and circumferential planes (Fig. 1E). The strain and 
A

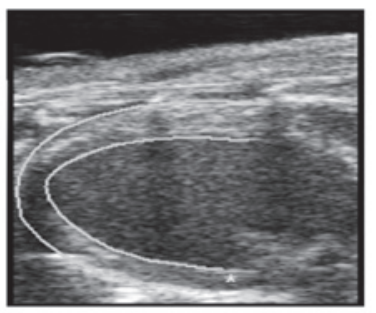

B

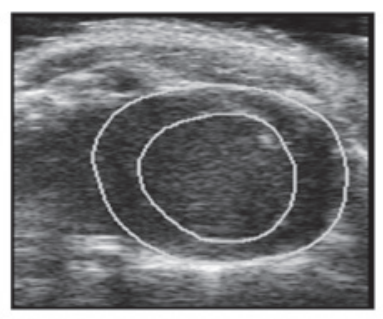

C

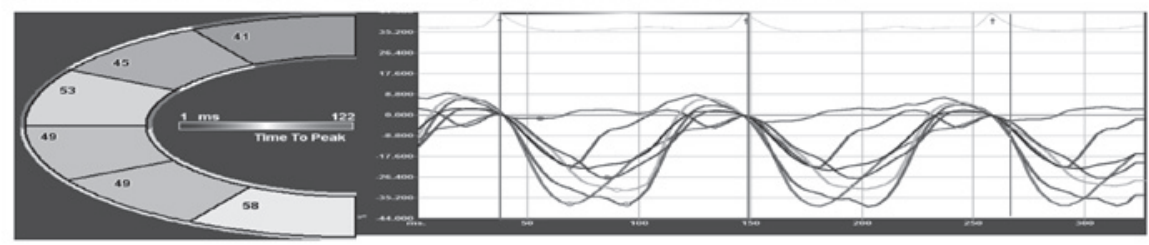

D

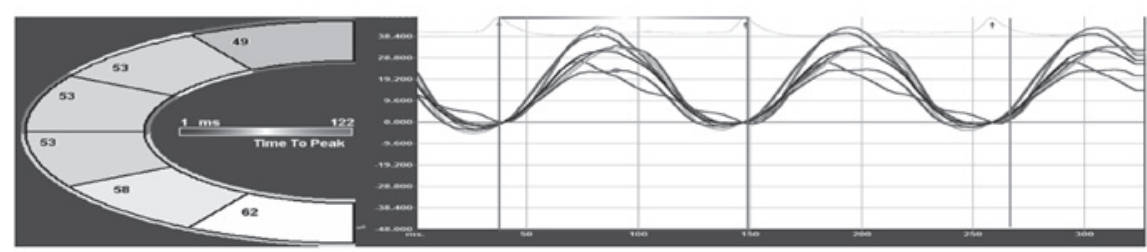

$\mathbf{E}$

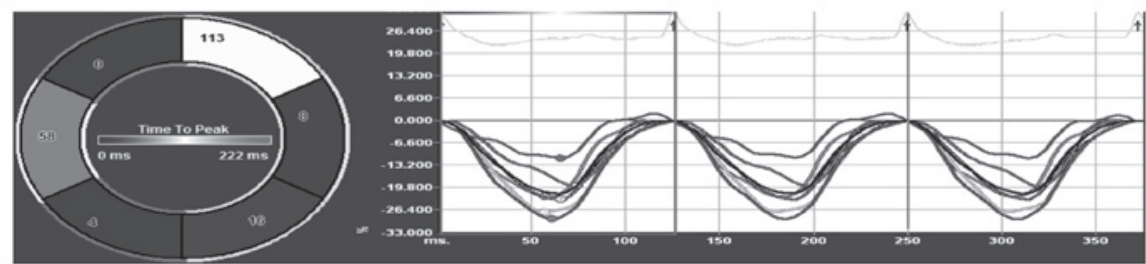

Figure 1. Representative images of the STE analysis. Endocardium and epicardium were semi-automated and traced by the analyzing software in parasternal (A) long axis view and (B) short axis view. (C) Longitudinal and (D) radial strain value in each segment (including posterior base, mid posterior, posterior apex, anterior base, mid anterior and anterior apex) of left ventricle. (E) Circumferential strain curves for each segment (including anterior free wall, lateral wall, posterior wall, inferior free wall, posterior septum wall and anterior septum wall) of left ventricle. STE, speckle tracking echocardiogram.

A

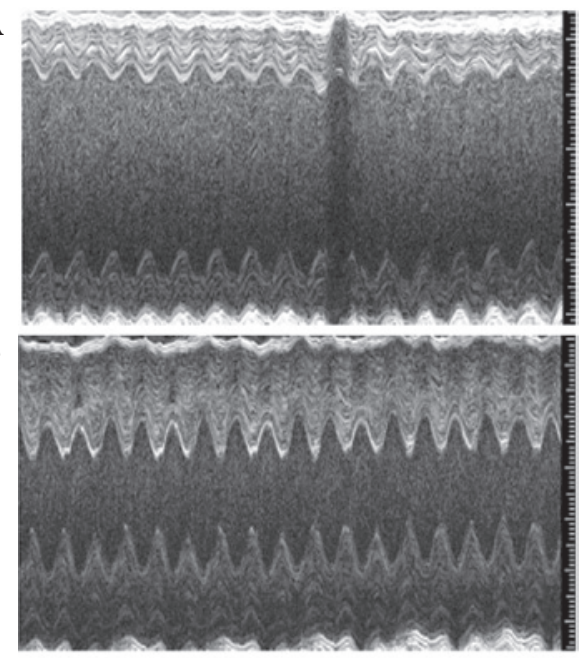

C

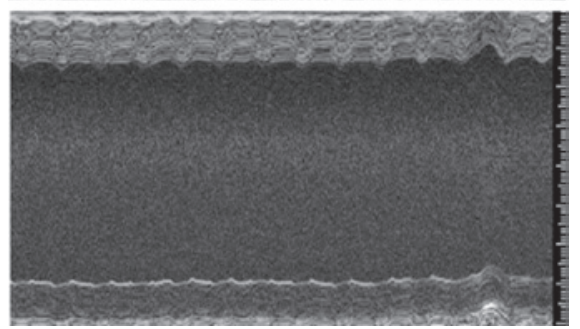

Figure 2. Typical short axis M-mode images of left ventricle in each group. Examples of short axis M-mode images from the (A) sham, (B) mild TAB and $(\mathrm{C})$ severe TAB groups. TAB, thoracic aortic banding. strain-rate of each segment were averaged in order to analyze global function.

Intra- and inter-observer variability. To assess the intra-observer variability, nine data sets from each group were selected at random and re-analyzed by the original investigator following an interval of several weeks. Inter-observer variability was assessed by comparing the results of two observers repeatedly analyzing the same data sets. Data were evaluated as the difference between the two observations divided by the mean and expressed as the percentage and the correlation coefficient $(15,16)$.

Statistical analysis. Data are expressed as the mean \pm standard error. The differences between the awake, LA and DA conditions were calculated using one-way analysis of variance, while Bonferroni post hoc analysis was used for multiple comparisons. $\mathrm{P}<0.05$ was considered to indicate a statistically significant difference. All analyses were performed using SPSS software, version 17.0 (SPSS, Inc., Chicago, IL, USA).

\section{Results}

Classic hypertrophic responses were observed in mice in the mild TAB group. The response included the preservation of EF and FS during the hypertrophic stage and a significant 
Table I. Postoperative changes in echocardiographic parameters following TAB.

\begin{tabular}{|c|c|c|c|}
\hline Parameter & Sham & Mild TAB & Severe TAB \\
\hline \multicolumn{4}{|l|}{ Conventional measures } \\
\hline Body weight (g) & $23.27 \pm 0.74$ & $27.06 \pm 0.35$ & $25.44 \pm 0.79$ \\
\hline HR (bpm) & $520 \pm 7^{\mathrm{ab}}$ & $512 \pm 6^{a}$ & $477 \pm 9$ \\
\hline LVEDD (mm) & $3.6 \pm 0.09^{\mathrm{a}}$ & $3.67 \pm 0.17^{\mathrm{a}}$ & $4.31 \pm 0.44$ \\
\hline $\operatorname{LVESD}(\mathrm{mm})$ & $2.09 \pm 0.09^{\mathrm{a}}$ & $2.05 \pm 0.23^{\mathrm{a}}$ & $3.14 \pm 0.60$ \\
\hline LVWT (mm) & $1.13 \pm 0.02^{\mathrm{b}}$ & $1.46 \pm 0.03^{\mathrm{a}}$ & $1.17 \pm 0.06$ \\
\hline LVEDV $(\mu \mathrm{l})$ & $55.23 \pm 2.87^{\mathrm{a}}$ & $60.96 \pm 6.60^{\mathrm{a}}$ & $100.57 \pm 21.88$ \\
\hline LVESV $(\mu 1)$ & $15.10 \pm 1.35^{\mathrm{a}}$ & $22.71 \pm 6.79^{a}$ & $70.72 \pm 23.05$ \\
\hline $\mathrm{EF}(\%)$ & $76.15 \pm 2.71^{\mathrm{a}}$ & $73.74 \pm 4.02^{\mathrm{a}}$ & $46.18 \pm 4.79$ \\
\hline $\mathrm{FS}(\%)$ & $46.75 \pm 2.47^{\mathrm{a}}$ & $41.36 \pm 3.61^{\mathrm{a}}$ & $22.10 \pm 3.92$ \\
\hline LV mass (mg) & $105.52 \pm 5.59^{\mathrm{ab}}$ & $159.04 \pm 11.30^{\mathrm{a}}$ & $198.88 \pm 12.87$ \\
\hline Corrected LV mass (mg) & $84.41 \pm 4.47^{\mathrm{ab}}$ & $133.27 \pm 10.21^{\mathrm{a}}$ & $161.10 \pm 10.30$ \\
\hline $\mathrm{SV}(\mu \mathrm{l})$ & $40.13 \pm 2.08^{\mathrm{a}}$ & $42.06 \pm 2.67^{\mathrm{a}}$ & $29.85 \pm 6.25$ \\
\hline \multicolumn{4}{|l|}{ Strain measures } \\
\hline \multicolumn{4}{|l|}{ Short axis } \\
\hline CS $(\%)$ & $-28.91 \pm 0.51^{\mathrm{a}}$ & $-29.15 \pm 1.44^{\mathrm{a}}$ & $-12.87 \pm 0.78$ \\
\hline $\operatorname{CSR}\left(\mathrm{s}^{-1}\right)$ & $-10.46 \pm 0.45^{\mathrm{a}}$ & $-10.68 \pm 0.8^{a}$ & $-4.74 \pm 0.06$ \\
\hline \multicolumn{4}{|l|}{ Long axis } \\
\hline $\operatorname{LS}(\%)$ & $-26.83 \pm 1.8^{\mathrm{ab}}$ & $-20.01 \pm 1.43^{\mathrm{a}}$ & $-15.4 \pm 0.94$ \\
\hline $\operatorname{LSR}\left(\mathrm{s}^{-1}\right)$ & $-10.99 \pm 0.34^{\mathrm{ab}}$ & $-7.8 \pm 0.41^{a}$ & $-5.7 \pm 0.21$ \\
\hline RS (\%) & $34.22 \pm 1.01^{\mathrm{ab}}$ & $26.77 \pm 0.12^{a}$ & $14.28 \pm 1.08$ \\
\hline $\operatorname{RSR}\left(\mathrm{s}^{-1}\right)$ & $9.95 \pm 0.62^{\mathrm{ab}}$ & $7.45 \pm 0.22^{\mathrm{a}}$ & $4.58 \pm 0.32$ \\
\hline
\end{tabular}

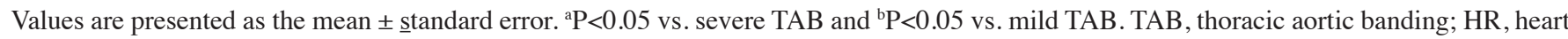
rate; LVWT, left ventricular wall thickness; LVEDD, left ventricular end-diastolic diameter; LVESD, left ventricular end-systolic diameter; LVESV, left ventricular end-systolic volume; LVEDV, left ventricular end-diastolic volume; EF, ejection fraction; FS, fraction shortening; LV mass; left ventricular mass; SV, stroke volume; CS, circumferential strain; CSR, circumferential strain rate; LS, longitudinal strain; LSR, longitudinal strain rate; RS, radial strain; RSR, radial strain rate.

increase in the LV wall thickness and mass compared with the sham group (Table I). The STE parameters, including longitudinal strain (LS), radial strain (RS) and corresponding strain rate exhibited a small reduction compared with the sham group. The circumferential strain (CS) and strain rate were preserved in the mild TAB group compared with the sham group (Table I), which may be attributed to the compensation mechanism (15). Furthermore, the severe TAB group mice exhibited a trend towards heart failure (HF) following TAB (Table I). In addition, severe TAB mice manifested typical clinical features of HF syndrome, including lethargy, impaired mobility and edema. During the post-banding period, 4/15 severe TAB mice succumbed, possibly to acute decompensated HF and arrhythmia. Three mice were excluded from the severe TAB group due to bradycardia. Conventional echocardiography indicated that EF and FS were significantly reduced in the severe TAB group (Table I). The STE parameters, including LS, RS, CS and corresponding strain rate were significantly reduced in the severe TAB group compared with the sham and mild TAB groups, whilst an increase was observed in the LVEDV and LVESV values (Table I). Fig. 2 exhibits marked differences in cardiac contraction and LV wall chamber cavity sizes among the sham, mild TAB and severe TAB groups.
Effects of anesthesia on conventional echocardiography. To assess the effect of anesthesia on conventional echocardiographic parameters, the values for these parameters in the awake, LA and DA conditions were compared among the sham (Table II), mild TAB (Table III) and severe TAB groups (Table IV). In all three groups the stepwise increase of ISF dose appeared to gradually reduce HR under the awake, LA and DA conditions $(\mathrm{P}<0.05)$. The conventional echocardiographic parameters examined included LVEDD, LVESD, LVPWTd, LVPWTs (LV posterior wall thickness in systole), LVAWTd, LVAWTs (LV anterior wall thickness in systole), LVEDV, LVESV, EF, FS, LV mass, corrected LV mass and SV. All of these parameters remained stable under the LA and awake conditions in the sham and mild TAB groups ( $\mathrm{P}>0.05$; Tables II and III). However, an increase in ISF concentration resulted in a significant suppression of these conventional parameters under the DA condition compared with the awake and LA conditions $(\mathrm{P}<0.05$; Tables II and III). However, there were exceptions, including the LV and corrected LV mass in the sham and mild TAB groups, which did not change with the different ISF conditions. Furthermore, no reduction was observed in the LVPWTd parameter in the sham group under DA conditions. Analysis of the severe TAB group indicated that all the conventional echocardiographic parameters remained constant under awake, LA and DA conditions (Table IV). 
Table II. Conventional echocardiographic parameters in the sham group mice under different states of anesthesia.

\begin{tabular}{lccc}
\hline Parameter & Awake & LA & DA \\
\hline Weight $(\mathrm{g})$ & $22.52 \pm 0.41$ & $23.27 \pm 0.74$ & $23.15 \pm 0.66$ \\
HR $(\mathrm{bpm})$ & $567 \pm 11^{\mathrm{ab}}$ & $520 \pm 5^{\mathrm{a}}$ & $407 \pm 10$ \\
LVEDD $(\mathrm{mm})$ & $3.51 \pm 0.07^{\mathrm{a}}$ & $3.6 \pm 0.09^{\mathrm{a}}$ & $4.01 \pm 0.10$ \\
LVESD $(\mathrm{mm})$ & $2.06 \pm 0.07^{\mathrm{a}}$ & $2.09 \pm 0.09^{\mathrm{a}}$ & $2.88 \pm 0.12$ \\
LVPWTd (mm) & $0.82 \pm 0.02$ & $0.81 \pm 0.03$ & $0.77 \pm 0.028$ \\
LVPWTs $(\mathrm{mm})$ & $1.31 \pm 0.04^{\mathrm{a}}$ & $1.40 \pm 0.06^{\mathrm{a}}$ & $1.08 \pm 0.044$ \\
LVAWTd $(\mathrm{mm})$ & $0.80 \pm 0.03^{\mathrm{a}}$ & $0.85 \pm 0.03^{\mathrm{a}}$ & $0.69 \pm 0.04$ \\
LVAWTs $(\mathrm{mm})$ & $1.34 \pm 0.04^{\mathrm{a}}$ & $1.35 \pm 0.04^{\mathrm{a}}$ & $1.00 \pm 0.06$ \\
LVEDV $(\mu l)$ & $52.41 \pm 2.27^{\mathrm{a}}$ & $55.23 \pm 2.87^{\mathrm{a}}$ & $70.791 \pm 4.00$ \\
LVESV $(\mu \mathrm{l})$ & $14.79 \pm 1.18^{\mathrm{a}}$ & $15.10 \pm 1.35^{\mathrm{a}}$ & $40.32 \pm 3.23$ \\
EF $(\%)$ & $72.53 \pm 1.52^{\mathrm{a}}$ & $76.15 \pm 2.71^{\mathrm{a}}$ & $54.34 \pm 2.55$ \\
FS $(\%)$ & $41.29 \pm 1.30^{\mathrm{a}}$ & $46.75 \pm 2.47^{\mathrm{a}}$ & $28.09 \pm 1.60$ \\
LV mass $(\mathrm{mg})$ & $96.79 \pm 3.43$ & $105.52 \pm 5.59$ & $84.00 \pm 5.72$ \\
Corrected LV mass $(\mathrm{mg})$ & $77.44 \pm 2.75$ & $84.41 \pm 4.47$ & $84.81 \pm 4.58$ \\
SV $(\mu l)$ & $37.62 \pm 1.57^{\mathrm{a}}$ & $40.13 \pm 2.08^{\mathrm{a}}$ & $27.48 \pm 1.93$ \\
\hline
\end{tabular}

Values are presented as the mean \pm standard error. ${ }^{a} \mathrm{P}<0.05$ vs. DA and ${ }^{\mathrm{b}} \mathrm{P}<0.05$ vs. LA. LA, light anesthesia; DA, deep anesthesia; HR, heart rate; LVEDD, left ventricular end-diastolic diameter; LVESD, left ventricular end-systolic diameter; LVPWTd, left ventricular posterior wall thickness in diastole; LVPWTs, left ventricular posterior wall thickness in systole; LVAWTd, left ventricular anterior wall thickness in diastole; LVAWTs, left ventricular anterior wall thickness in systole; LVESV, left ventricular end-systolic volume; LVEDV, left ventricular end-diastolic volume; EF, ejection fraction; FS, fraction shortening; LV mass; left ventricular mass; SV, stroke volume.

Table III. Conventional echocardiographic parameters in the mild TAB group mice under different states of anesthesia.

\begin{tabular}{lcrc}
\hline Parameter & Awake & LA & DA \\
\hline Weight $(\mathrm{g})$ & $27.23 \pm 0.21$ & $27.06 \pm 0.35$ & $26.47 \pm 0.77$ \\
HR $(\mathrm{bpm})$ & $563 \pm 4^{\mathrm{ab}}$ & $512 \pm 6^{\mathrm{a}}$ & $395 \pm 8$ \\
LVEDD $(\mathrm{mm})$ & $3.53 \pm 0.10^{\mathrm{a}}$ & $3.67 \pm 0.17^{\mathrm{a}}$ & $4.44 \pm 0.08$ \\
LVESD $(\mathrm{mm})$ & $1.88 \pm 0.13^{\mathrm{a}}$ & $2.05 \pm 0.23^{\mathrm{a}}$ & $3.46 \pm 0.14$ \\
LVPWTd $(\mathrm{mm})$ & $1.13 \pm 0.04^{\mathrm{a}}$ & $1.09 \pm 0.02^{\mathrm{a}}$ & $0.93 \pm 0.04$ \\
LVPWTs $(\mathrm{mm})$ & $1.67 \pm 0.06^{\mathrm{a}}$ & $1.59 \pm 0.09^{\mathrm{a}}$ & $1.12 \pm 0.07$ \\
LVAWTd $(\mathrm{mm})$ & $1.24 \pm 0.05^{\mathrm{a}}$ & $1.13 \pm 0.05^{\mathrm{a}}$ & $0.94 \pm 0.05$ \\
LVAWTs $(\mathrm{mm})$ & $1.88 \pm 0.07^{\mathrm{a}}$ & $1.74 \pm 0.08^{\mathrm{a}}$ & $1.27 \pm 0.08$ \\
LVEDV $(\mu \mathrm{l})$ & $52.85 \pm 3.48^{\mathrm{a}}$ & $60.96 \pm 6.60^{\mathrm{a}}$ & $89.70 \pm 3.75$ \\
LVESV $(\mu \mathrm{l})$ & $12.33 \pm 2.23^{\mathrm{a}}$ & $22.71 \pm 6.79^{\mathrm{a}}$ & $51.29 \pm 4.82$ \\
EF $(\%)$ & $78.88 \pm 2.53^{\mathrm{a}}$ & $73.74 \pm 4.02^{\mathrm{a}}$ & $44.45 \pm 3.49$ \\
FS $(\%)$ & $47.96 \pm 2.29^{\mathrm{a}}$ & $41.36 \pm 3.61^{\mathrm{a}}$ & $22.47 \pm 2.19$ \\
LV mass $(\mathrm{mg})$ & $172.86 \pm 9.33$ & $159.04 \pm 11.30$ & $175.02 \pm 7.72$ \\
Corrected LV mass $(\mathrm{mg})$ & $138.37 \pm 7.47$ & $133.27 \pm 10.21$ & $140.04 \pm 6.18$ \\
SV $(\mu l)$ & $40.53 \pm 2.06^{\mathrm{a}}$ & $42.06 \pm 2.6^{\mathrm{a}}$ & $31.41 \pm 2.01$ \\
\hline
\end{tabular}

Values are presented as the mean \pm standard error. ${ }^{\text {a }}<0.05$ vs. DA and ${ }^{\mathrm{b}} \mathrm{P}<0.05$ vs. LA. TAB, thoracic aortic banding; LA, light anesthesia; DA, deep anesthesia; HR, heart rate; LVEDD, left ventricular end-diastolic diameter; LVESD, left ventricular end-systolic diameter; LVPWTd, left ventricular posterior wall thickness in diastole; LVPWTs, left ventricular posterior wall thickness in systole; LVAWTd, left ventricular anterior wall thickness in diastole; LVAWTs, left ventricular anterior wall thickness in systole; LVESV, left ventricular end-systolic volume; LVEDV, left ventricular end-diastolic volume; EF, ejection fraction; FS, fraction shortening; LV mass; left ventricular mass; SV, stroke volume.

Effects of anesthesia on STE. The strain and corresponding strain rates were acquired from mice under the awake, LA and DA states in order to determine the effect of anesthesia on the results of STE examination. No significant differences in LS, CS, RS or corresponding strain rates were observed between the LA and awake conditions in the three groups 
Table IV. Conventional echocardiographic parameters in the severe TAB group mice under different states of anesthesia.

\begin{tabular}{lccc}
\hline Parameter & Awake & LA & DA \\
\hline Weight $(\mathrm{g})$ & $27.16 \pm 1.27$ & $25.44 \pm 0.79$ & $24.35 \pm 0.47$ \\
HR $(\mathrm{bpm})$ & $523 \pm 5^{\mathrm{ab}}$ & $477 \pm 9^{\mathrm{a}}$ & $404 \pm 8$ \\
LVEDD $(\mathrm{mm})$ & $4.54 \pm 0.47$ & $4.31 \pm 0.44$ & $4.95 \pm 0.28$ \\
LVESD $(\mathrm{mm})$ & $3.60 \pm 0.61$ & $3.14 \pm 0.60$ & $4.09 \pm 0.43$ \\
LVPWTd $(\mathrm{mm})$ & $0.99 \pm 0.12$ & $0.96 \pm 0.11$ & $0.84 \pm 0.04$ \\
LVPWTs $(\mathrm{mm})$ & $1.29 \pm 0.17$ & $1.40 \pm 0.17$ & $1.04 \pm 0.04$ \\
LVAWTd $(\mathrm{mm})$ & $1.16 \pm 0.06$ & $1.13 \pm 0.06$ & $1.02 \pm 0.05$ \\
LVAWTs $(\mathrm{mm})$ & $1.46 \pm 0.11$ & $1.49 \pm 0.10$ & $1.32 \pm 0.11$ \\
LVEDV $(\mu \mathrm{l})$ & $101.30 \pm 23.73$ & $100.57 \pm 21.88$ & $103.03 \pm 14.44$ \\
LVESV $(\mu \mathrm{l})$ & $75.66 \pm 23.74$ & $70.72 \pm 23.05$ & $75.16 \pm 18.14$ \\
EF $(\%)$ & $47.03 \pm 11.23$ & $46.18 \pm 4.79$ & $46.84 \pm 7.93$ \\
FS $(\%)$ & $26.42 \pm 8.36$ & $22.10 \pm 3.92$ & $28.44 \pm 4.37$ \\
LV mass $(\mathrm{mg})$ & $209.36 \pm 12.13$ & $198.88 \pm 12.87$ & $204.42 \pm 20.13$ \\
Corrected LV mass $(\mathrm{mg})$ & $167.48 \pm 9.71$ & $161.10 \pm 10.30$ & $163.53 \pm 16.11$ \\
SV $(\mu l)$ & $28.64 \pm 7.55$ & $29.85 \pm 6.25$ & $27.87 \pm 7.16$ \\
\hline
\end{tabular}

Values are presented as the mean \pm standard error. ${ }^{\mathrm{a}} \mathrm{P}<0.05$ vs. DA and ${ }^{\mathrm{b}} \mathrm{P}<0.05$ vs. LA. TAB, thoracic aortic banding; LA, light anesthesia; $\mathrm{DA}$, deep anesthesia; HR, heart rate; LVEDD, left ventricular end-diastolic diameter; LVESD, left ventricular end-systolic diameter; LVPWTs, left ventricular posterior wall thickness in systole; LVPWTd, left ventricular posterior wall thickness in diastole; LVAWTd, left ventricular anterior wall thickness in diastole; LVAWTs, left ventricular anterior wall thickness in systole; LVESV, left ventricular end-systolic volume; LVEDV, left ventricular end-diastolic volume; EF, ejection fraction; FS, fraction shortening; LV mass; left ventricular mass; SV, stroke volume.

A

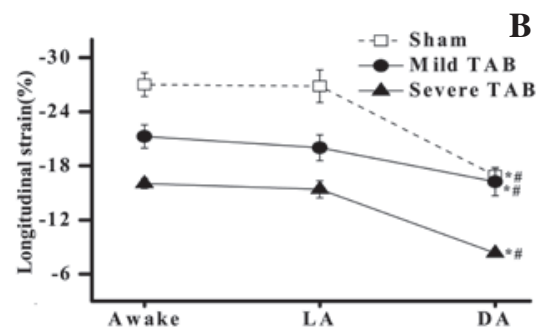

D

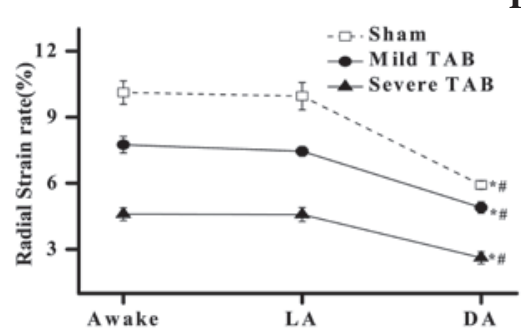

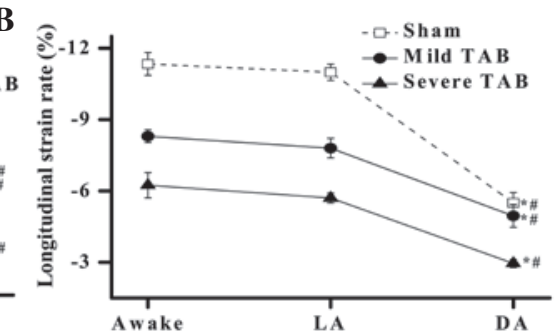

$\mathbf{E}$

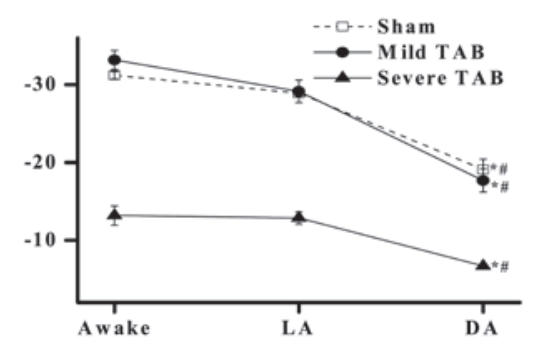

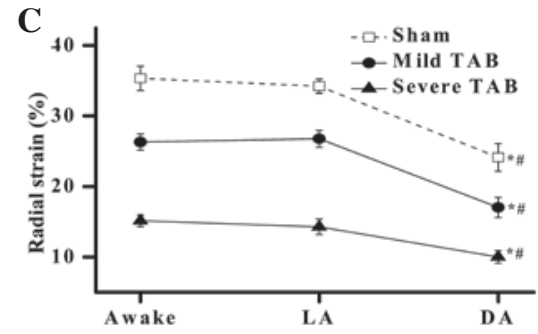

F

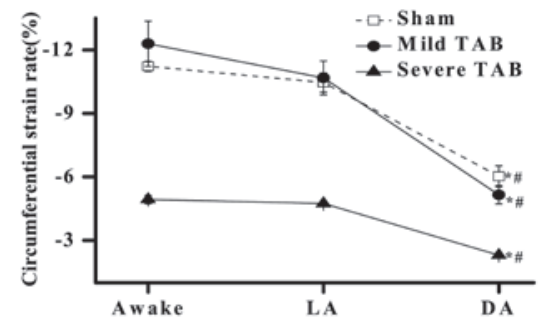

Figure 3. Effect of anesthesia on STE parameters in the sham, mild TAB and severe TAB groups. Comparison of (A) longitudinal strain and (B) corresponding strain rate under awake, LA and DA conditions. Analysis of (C) radial strain and (D) corresponding strain rate under awake, LA and DA conditions. Evaluation of (E) circumferential strain and (F) corresponding strain rate under awake, LA and DA conditions. "P<0.05 vs. awake and " P<0.05 vs. LA. STE, speckle tracking echocardiogram; TAB, thoracic aortic banding; LA, light anesthesia; DA, deep anesthesia.

(P>0.05; Fig. 3). However, the STE parameters in all three groups exhibited significant differences under DA conditions compared with LA and awake conditions $(\mathrm{P}<0.05$; Fig. 3$)$.

Inter- and intra-observer variability. The results of the analysis of inter- and intra-observer variability are presented in Table V. The data indicated no significant level of inter- and intra-observer variability and very high correlation coefficients for the conventional and STE parameters.

\section{Discussion}

The present study demonstrated that under LA conditions (HR range, 460-520 bpm; ISF range, 0.5-1\%), conventional echocardiography and STE may be applied in healthy, hypertrophic and HF myocardium with a slight reduction of cardiac function.

Previous studies have reported that echocardiographic parameters are affected by anesthetic agents and that varying doses of anesthesia may influence the result of echocardio- 
Table V. Intra- and inter-observer variability for conventional and speckle tracking echocardiography.

\begin{tabular}{|c|c|c|c|c|}
\hline \multirow[b]{2}{*}{ Parameter } & \multicolumn{2}{|c|}{ Inter-observer } & \multicolumn{2}{|c|}{ Intra-observer } \\
\hline & $\%$ Error & $\mathrm{CC}$ & $\%$ Error & $\mathrm{CC}$ \\
\hline \multicolumn{5}{|l|}{ Conventional measures } \\
\hline LVEDD (mm) & $0.7 \pm 1.3$ & 0.84 & $0.5 \pm 0.2$ & 0.86 \\
\hline LVESD (mm) & $3.3 \pm 2.6$ & 0.98 & $2.4 \pm 1.7$ & 0.99 \\
\hline LVPWTd (mm) & $9.6 \pm 2.5$ & 0.89 & $4.3 \pm 1.8$ & 0.94 \\
\hline LVPWTs (mm) & $3.8 \pm 2.6$ & 0.95 & $4.5 \pm 1.4$ & 0.92 \\
\hline LVAWTd (mm) & $4.9 \pm 1.1$ & 0.89 & $3.7 \pm 1.3$ & 0.9 \\
\hline LVAWTs (mm) & $2.6 \pm 3.1$ & 0.97 & $1.7 \pm 3.5$ & 0.98 \\
\hline $\operatorname{LVEDV}(\mu \mathrm{l})$ & $1.6 \pm 3.0$ & 0.92 & $1.4 \pm 3.2$ & 0.91 \\
\hline LVESV $(\mu 1)$ & $4.6 \pm 0.9$ & 0.80 & $3.5 \pm 0.7$ & 0.82 \\
\hline $\mathrm{EF}(\%)$ & $6.2 \pm 1.5$ & 0.82 & $4.9 \pm 0.5$ & 0.87 \\
\hline $\mathrm{FS}(\%)$ & $5.7 \pm 1.2$ & 0.87 & $7.8 \pm 0.6$ & 0.89 \\
\hline LV mass (mg) & $8.2 \pm 4.6$ & 0.83 & $5.6 \pm 1.7$ & 0.87 \\
\hline Corrected LV mass (mg) & $8.2 \pm 4.6$ & 0.83 & $4.4 \pm 6.2$ & 0.91 \\
\hline $\mathrm{SV}(\mu \mathrm{l})$ & $0.7 \pm 1.8$ & 0.82 & $0.9 \pm 1.4$ & 0.85 \\
\hline \multicolumn{5}{|l|}{ Strain measures } \\
\hline \multicolumn{5}{|l|}{ Short axis } \\
\hline CS (\%) & $1.8 \pm 1.2$ & 0.97 & $1.4 \pm 0.3$ & 0.98 \\
\hline $\operatorname{CSR}\left(\mathrm{s}^{-1}\right)$ & $4.2 \pm 4.1$ & 0.94 & $5.9 \pm 4.5$ & 0.92 \\
\hline \multicolumn{5}{|l|}{ Long axis } \\
\hline $\operatorname{LS}(\%)$ & $1.9 \pm 1.6$ & 0.92 & $1.8 \pm 2.1$ & 0.90 \\
\hline $\operatorname{LSR}\left(\mathrm{s}^{-1}\right)$ & $3.8 \pm 3.0$ & 0.93 & $4.5 \pm 4.0$ & 0.96 \\
\hline $\mathrm{RS}(\%)$ & $2.5 \pm 1.6$ & 0.90 & $3.8 \pm 1.9$ & 0.91 \\
\hline $\operatorname{RSR}\left(\mathrm{s}^{-1}\right)$ & $3.2 \pm 2.6$ & 0.97 & $2.3 \pm 1.9$ & 0.99 \\
\hline
\end{tabular}

Values are presented as the mean \pm standard error. LVEDD, left ventricular end-diastolic diameter; LVESD, left ventricular end-systolic diameter; LVPWTd, left ventricular posterior wall thickness in diastole; LVPWTs, left ventricular posterior wall thickness in systole; LVAWTd, left ventricular anterior wall thickness in diastole; LVAWTs, left ventricular anterior wall thickness in systole; LVESV, left ventricular end-systolic volume; LVEDV, left ventricular end-diastolic volume; EF, ejection fraction; FS, fraction shortening; SV, stroke volume; CS, circumferential strain; LS, longitudinal strain; RS, radial strain; LSR, longitudinal strain rate; CSR, circumferential strain rate; RSR, radial strain rate.

graphic measurement (16-18). In the present study, a marked reduction was observed in the conventional echocardiographic parameters of the sham group under DA conditions. However, these appeared to remain constant under awake and LA conditions. In agreement with this, conventional echocardiographic parameters were highly repeatable in the present study under LA conditions, despite a slight elevation in ISF dose and HR.

Anesthesia is considered to be an influential factor in the measurement of conventional echocardiographic parameters in healthy myocardium. However, a limited number of studies have investigated the effects of anesthesia on hypertrophic and failing myocardium. In the present study, conventional echocardiographic parameters in hypertrophic myocardium were reduced under DA conditions, but preserved under LA and awake conditions. However, the conventional echocardiographic parameters were unchanged in the failing myocardium under LA, DA and awake conditions. This may be attributable to the limited number of mice, which resulted in a large variability in conventional echocardiographic parameters in the severe TAB group compared with the sham and mild TAB groups.
The underlying mechanism for the improved reproducibility in mice under LA conditions may be attributed to the high HR in the LA and awake conditions. Generally, force-frequency dependence varied only minimally in high HR (>550 bpm) but altered significantly in the low HR conditions $(<400 \mathrm{bpm})$ in murine hearts. Therefore, LV contractility remained almost unaltered under high HR conditions, which explained why echocardiographic parameters were constant under LA conditions with high HR (18). The aforementioned data supports the hypothesis that conventional echocardiographic evaluation is clinically applicable under LA conditions in mice, with reduced cardiac systolic depression and perfect reproducibility. This protocol may be used in healthy or diseased mice.

The present study demonstrated that an increase of ISF dose and subsequent suppression of HR resulted in an evident reduction of the strain parameters in mice under DA conditions, but no alteration in mice in the LA and awake states. However, a previous study by Weidemann et al (19) indicated that strain increased following a reduction in HR. This contradiction may be explained by the following mechanism: Strain is determined primarily by ejection performance parameters 
such as SV $(20,21)$. Following a reduction in HR, the preload and ejection period increased, which led to an increase in SV and strain. However, under DA conditions, the increased administration of ISF reversed this trend by significantly reducing cardiac contraction $(22,23)$. Due to the abnormal contraction observed in mice under DA conditions, the SV was considerably suppressed, which resulted in a reduction in strain.

In addition, the effect of anesthesia on strain rate was analyzed. Increasing the ISF dose and thus reducing HR resulted in a reduction in strain rate in mice under DA conditions. However, strain rate remained constant in mice in the LA and awake states in each group. However, previous studies involving rats and dogs have indicated that strain rate is independent of HR $(14,24)$. This paradox may be explained by the following mechanism: With the reduction of HR, the intrinsic contractility reduced due to the negative Treppe effect (25). However, as the preload and ejection period increased, so did the intrinsic contractility, via the negative Frank-Starling mechanism (26). The combination of these two factors may have resulted in a balancing effect on the extent of contractility. Therefore, the strain rate as an index of change of contractility, remained the same as the HR fluctuated. However, as the dose of ISF increased under DA conditions, the contractility was suppressed significantly and the balancing effect on contractility was prevented. This may explain the significant reduction of strain rate under DA conditions.

There were a number of limitations to the present study: Firstly, conscious mice were not included as a control, as the elevation of sympathetic tone during conscious measurement could not be fully eliminated, despite the mice undergoing training a few days prior to the experiment (27). In addition, the images used for strain analysis must be of high resolution, with clear visualization of the endocardium and epicardium borders. Therefore, it is necessary that the mice be immobile, which would not have been feasible if the mice had remained conscious. Secondly, Doppler parameters were not measured due to the high HR, as Doppler waveforms merge together when there is a reduction in the diastolic period $(28,29)$. Thirdly, bradycardic mice with a low HR $(<500 \mathrm{bpm})$ and HF in the severe $\mathrm{TAB}$ group were excluded from the study. Therefore, the least healthy mice with HF were omitted from the study and the effects of anesthesia on HF mice may have been underestimated.

In conclusion, the present study conducted a novel investigation into the effects of anesthesia on conventional echocardiography and STE parameters in healthy mice and pathological models. The pathological model is of particular clinical relevance, as anesthesia may severely reduce cardiac contraction in patients with HF. In addition, the present study examined the effects of anesthesia in a mouse model of pressure overload/aortic stenosis and assessed whether an anesthetic was able to limit the effectiveness of echocardiography in the evaluation of cardiac function (30). Under LA conditions, relatively precise conventional and STE measurements may be obtained without a significant reduction in cardiac function. This approach offers a potentially valuable method for accurately measuring conventional and STE parameters with good reproducibility. Thus, immobility and sedation may widen the applicability of conventional echocardiography and STE in healthy and diseased mice.

\section{Acknowledgements}

The current study was supported by grants from the Hubei Science \& Technology Pillar Program (no. 2012DCA12007) and the National Natural Science Foundation of China (no. 81300141).

\section{References}

1. Yu L, Ruifrok WP, Meissner M, et al: Genetic and pharmacological inhibition of galectin-3 prevents cardiac remodeling by interfering with myocardial fibrogenesis. Circ Heart Fail 6: 107-117, 2013.

2. Lairez O, Calise D, Bianchi P, et al: Genetic deletion of MAO-A promotes serotonin-dependent ventricular hypertrophy by pressure overload. J Mol Cell Cardiol 46: 587-595, 2009.

3. Asai K, Yang GP, Geng YJ, et al: Beta-adrenergic receptor blockade arrests myocyte damage and preserves cardiac function in the transgenic G(salpha) mouse. J Clin Invest 104: 551-558, 1999.

4. Guellich A, Gao S, Hong C, et al: Effects of cardiac overexpression of type 6 adenylyl cyclase affects on the response to chronic pressure overload. Am J Physiol Heart Circ Physiol 299: H707-H712, 2010.

5. Stanton T and Marwick TH: Assessment of subendocardial structure and function. JACC Cardiovasc Imaging 3: 867-875, 2010.

6. Cottrell C and Kirkpatrick JN: Echocardiographic strain imaging and its use in the clinical setting. Expert Rev Cardiovasc Ther 8: 93-102, 2010.

7. Abduch MC, Alencar AM, Mathias W Jr and Vieira ML: Cardiac mechanics evaluated by speckle tracking echocardiography. Arq Bras Cardiol 4: 403-412, 2014.

8. Kurt M, Tanboga IH and Aksakal E: Two-dimensional strain imaging: Basic principles and technical consideration. Eurasian J Med 46: 126-130, 2014.

9. Roth DM, Swaney JS, Dalton ND, Gilpin EA and Ross J Jr.: Impact of anesthesia on cardiac function during echocardiography in mice. Am J Physiol Heart Circ Physiol 282: H2134-H2140, 2002.

10. Collins KA, Korcarz CE and Lang RM: Use of echocardiography for the phenotypic assessment of genetically altered mice. Physiol Genomics 13: 227-239, 2003.

11. Lairez O, Lonjaret L, Ruiz S, et al: Anesthetic regimen for cardiac function evaluation by echocardiography in mice: comparison between ketamine, etomidate and isoflurane versus conscious state. Lab Anim 47: 284-290, 2013.

12. Szczesny G, Veihelmann A, Massberg S, Nolte D and Messmer K: Long-term anaesthesia using inhalatory isoflurane in different strains of mice-the haemodynamic effects. Lab Anim 38: 64-69, 2004.

13. Matsuda Y, Ohsaka K, Yamamoto H, et al: Comparison of newly developed inhalation anesthesia system and intraperitoneal anesthesia on the hemodynamic state in mice. Biol Pharm Bull 30: 1716-1720, 2007.

14. Weytjens C, D'Hooge J, Droogmans S, et al: Influence of heart rate reduction on Doppler myocardial imaging parameters in a small animal model. Ultrasound Med Biol 35: 30-35, 2009.

15. Tanaka N, Dalton N, Mao L, et al: Transthoracic echocardiography in models of cardiac disease in the mouse. Circulation 94: 1109-1117, 1996.

16. Gentry-Smetana S, Redford D, Moore D and Larson DF: Direct effects of volatile anesthetics on cardiac function. Perfusion 23: 43-47, 2008.

17. Takamura $\mathrm{T}$, Dohi $\mathrm{K}$, Onishi $\mathrm{K}$, et al: Left ventricular contraction-relaxation coupling in normal, hypertrophic, and failing myocardium quantified by speckle-tracking global strain and strain rate imaging. J Am Soc Echocardiogr 23: 747-754, 2010.

18. Riha H, Papoušek F, Neckář J, Pirk J and Ošt’ádal B: Effects of isoflurane concentration on basic echocardiographic parameters of the left ventricle in rats. Physiol Res 61: 419-423, 2012.

19. Weidemann F, Jamal F, Kowalski M, et al: Can strain rate and strain quantify changes in regional systolic function during dobutamine infusion, B-blockade, and atrial pacing - implications for quantitative stress echocardiography. J Am Soc Echocardiogr 15: 416-424, 2002.

20. Morris JJ III, Pellom GL, Murphy CE, Salter DR, Goldstein JP and Wechsler AS: Quantification of the contractile response to injury: assessment of the work-length relationship in the intact heart. Circulation 76: 717-727, 1987. 
21. Weidemann F, Jamal F, Sutherland GR, et al: Myocardial function defined by strain rate and strain during alterations in inotropic states and heart rate. Am J Physiol Heart Circ Physiol 283 H792-H799, 2002

22. Davies LA, Gibson CN, Boyett MR, Hopkins PM and Harrison SM: Effects of isoflurane, sevoflurane, and halothane on myofilament $\mathrm{Ca}^{2+}$ sensitivity and sarcoplasmic reticulum $\mathrm{Ca}^{2+}$ release in rat ventricular myocytes. Anesthesiology 93: 1034-1044, 2000.

23. Davies LA, Hamilton DL, Hopkins PM, Boyett MR and Harrison SM: Concentration-dependent inotropic effects of halothane, isoflurane and sevoflurane on rat ventricular myocytes. Br J Anaesth 82: 723-730, 1999.

24. Suzuki R, Matsumoto H, Teshima $T$ and Koyama H: Influence of heart rate on myocardial function using two-dimensional speckle-tracking echocardiography in healthy dogs. J Vet Cardiol 15: 139-146, 2013.

25. Woodworth RS: Maximal contraction, "staircase" contraction, refractory period, and compensatory pause of the heart. Am J Physiol 8: 213-249, 1902.
26. Katz AM: Ernest Henry Starling, his predecessors, and the "Law of the Heart". Circulation 106: 2986-2992, 2002.

27. Uechi M, Asai K, Osaka M, et al: Depressed heart rate variability and arterial baroreflex in conscious transgenic mice with overexpression of cardiac Gsalpha. Circ Res 82: 416-423, 1998.

28. Schaefer A, Meyer GP, Brand B, Hilfiker-Kleiner D, Drexler H and Klein G: Effects of anesthesia on diastolic function in mice assessed by echocardiography. Echocardiography 22: 665-670, 2005.

29. Rottman JN, Ni G, Khoo M, et al: Temporal changes in ventricular function assessed echocardiographically in conscious and anesthetized mice. J Am Soc Echocardiogr 16: 1150-1157, 2003.

30. Weiss RM, Ohashi M, Miller JD, Young SG and Heistad DD: Calcific aortic valve stenosis in old hypercholesterolemic mice. Circulation 114: 2065-2069, 2006. 